\title{
Coming out of the PiTs-novel strategies for controlling intestinal phosphate absorption in patients with CKD
}

\author{
Wagner, Carsten A
}

\begin{abstract}
The kidneys are the gatekeepers of phosphate balance, and loss of kidney function causes a profound disturbance of mineral metabolism. Patients with chronic kidney disease suffer from an excessive cardiovascular disease risk with a high morbidity and mortality. Current therapies aimed at reducing total phosphate body load are insufficient, and novel strategies are urgently needed. In this issue, Tsuboi and colleagues provide evidence for the use of a novel phosphate transporter inhibitor to reduce intestinal phosphate absorption.
\end{abstract}

DOI: https://doi.org/10.1016/j.kint.2020.04.010

Posted at the Zurich Open Repository and Archive, University of Zurich

ZORA URL: https://doi.org/10.5167/uzh-200199

Journal Article

Accepted Version

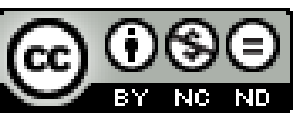

The following work is licensed under a Creative Commons: Attribution-NonCommercial-NoDerivatives 4.0 International (CC BY-NC-ND 4.0) License.

Originally published at:

Wagner, Carsten A (2020). Coming out of the PiTs-novel strategies for controlling intestinal phosphate absorption in patients with CKD. Kidney International, 98(2):273-275.

DOI: https://doi.org/10.1016/j.kint.2020.04.010 
Coming out of the PiTs - novel strategies for controlling intestinal phosphate absorption in patients with CKD

\author{
Carsten A Wagner \\ Institute of Physiology, University of Zurich and National Center of Competence \\ in Research NCCR Kidney.CH, Switzerland
}




\section{ABSTRACT}

The kidneys are the gate-keepers of phosphate balance and loss of kidney function causes a profound disturbance of mineral metabolism. Patients with CKD suffer from an excessive cardiovascular disease risk with a high morbidity and mortality. Current therapies aiming at reducing phosphate body phosphate load are insufficient and novel strategies urgently needed. In this issue, Tsuboi and colleagues provide evidence for the use of a novel phosphate transporter inhibitor to reduce intestinal phosphate absorption.

\section{Main text}

Phosphate is an essential mineral. A complex system regulating phosphate intake, excretion and its balance has evolved over millions of years of evolution. The kidneys play a central role in these mechanisms as the organ controlling excretion of phosphate but also affecting intestinal absorption of phosphate through the action of calcitriol synthesized in kidney proximal tubules and stimulating active transport of phosphate in the small intestine. The importance of the kidneys in this delicate balance becomes even more evident in patients with impaired kidney function. Disordered mineral balance is among the first signs of reduced kidney function when first calcitriol and $\alpha$-klotho levels fall followed by a rise in FGF23, and later in PTH and serum phosphate ${ }^{1-3}$.

Very little is known on the role of intestinal phosphate absorption in phosphate overload in patients with CKD. While it is beyond doubt that intestinal phosphate absorption is required for phosphate overload, less certainty exists whether or not intestinal phosphate absorption adapts to the state of CKD.

Intestinal phosphate absorption proceeds through at least two distinct pathways, a transporter-mediated transcellular pathway and a paracellular route 
4. The sodium-driven phosphate cotransporter NaPi-Ilb (Npt2b, SLC34A2) has been considered as the major intestinal phosphate transporter. It is located at the luminal side of enterocytes and mediates the first step in transcellular phosphate absorption. In humans (and rats) it is mostly found in the upper small intestine (i.e. duodenum and jejunum) whereas in mice NaPi-llb is expressed predominately in the lower small intestine (late jejunum and ileum). Humans carrying inactivating mutations in SLC34A2 develop pulmonary alveolar microlithiasis (PAM), a fibrosing lung disease caused by calcium phosphate crystals. However, no disturbances of systemic phosphate balance have been reported in PAM patients suggesting that the role of $\mathrm{NaPi}-\mathrm{Ilb}$ in intestinal phosphate absorption might be dispensable. Along the same line, genetic ablation of $\mathrm{NaPi}$-llb in mice abolishes active sodium-dependent phosphate absorption in ileum but systemic phosphate balance is maintained ${ }^{5}$. Only when dietary phosphate is restricted, the absence of NaPi-llb in the intestine causes loss of bone mass, presumably to provide phosphate to metabolism ${ }^{6}$.

This raises the question, which other pathways for intestinal phosphate absorption exist and how phosphate balance can be maintained in the absence of NaPi-Ilb in humans and mice. On possibility is an important contribution of paracellular phosphate fluxes to overall intestinal phosphate absorption. Indeed, human and rodent small intestine show a high paracellular phosphate flux when intestinal phosphate content is high, as present in typical diets in industrialized countries. This paracellular phosphate flux shows a high capacity, is probably not very much regulated or adapted to systemic phosphate requirements, and allows for large quantities of phosphate to be absorbed under conditions of high dietary phosphate availability. Another possibility is the presence of additional phosphate transporters contributing to transcellular phosphate transport. Indeed, the two members of the SLC20 family of sodium-dependent phosphate transporters PiT1 and PIT-2 are also expressed along the entire small and large intestine. However, until to date their contribution to intestinal phosphate absorption has been considered to be rather negligible. 
Several established therapies as well as therapies currently in clinical test phases target intestinal phosphate absorption ${ }^{3,} 7$. While dietary phosphate restriction and several classes of phosphate binders aim at reducing available phosphate, novel therapies try decreasing trans- and paracellular phosphate absorption. The specific NaPi-Ilb inhibitor ASP-3325 and nicotinamide reduce activity and/or abundance of NaPi-Ilb in the intestine. Nicotinamide may also decrease renal NaPi-Ila expression. However, ASP-3325 given alone completely failed to alter serum phosphate levels, FGF23 or intestinal phosphate absorption in patients on hemodialysis. Nicotinamide alone has been shown to reduce serum phosphate levels in some small studies, the COMBINE study comparing nicotinamide or a phosphate binder alone in combination vs placebo in 205 predialysis patients failed to show effects of any of these treatments on serum phosphate and FGF23 levels. In contrast, in patients on hemodialysis nicotinamide together with phosphate binders was able to reduce serum phosphate (unpublished data from NoPhos phase III trial as shown during ERAEDTA congress 2019 and discussed here ${ }^{7}$ ) as compared to patients receiving only standard care (including phosphate binders) ${ }^{7}$.

A different mode of action is used by Tenapanor, an inhibitor of the sodium-proton exchanger isoform $3\left(\mathrm{Na}^{+} / \mathrm{H}^{+}\right.$-exchanger $\left.\mathrm{NHE3}\right)$, that reduces intestinal $\mathrm{Na}^{+}$-absorption. The drug leads due to NHE3 inhibition to intracellular acidification, that in turn reduces paracellular phosphate fluxes ${ }^{8}$. Small studies in patients on hemodialysis have shown that tenapanor can reduce serum phosphate and FGF23 levels but whether tenapanor may be clinically useful remains to be established in larger studies as inhibition of NHE3 can also induce diarrhea. Nevertheless, tenapanor represents the only drug that is able to reduce paracellular phosphate absorption and it may thus be interesting to test tenapanor in combination with drugs reducing overall phosphate availability and/or transcellular phosphate absorption. 
Now, in a new study in this issue of Kidney International, Tsuboi and colleagues report the development of novel pan-phosphate transporter inhibitor (EOS-789) by Chugai Pharmaceuticals and its testing in normal rats and rats with impaired kidney function ${ }^{9}$. EOS-789 blocks in vitro all three isoforms of the SLC34 transporter family (i.e. NaPi-Ila, -Ilb, and -Ilc) as well as both isoforms of the PiT/SLC20 family (PiT-1, and PiT-2) with half-maximal inhibition in the low micromolar range. When given orally, systemic absorption appears to be low so that the effect of EOS-789 is likely confined only to phosphate transporters expressed at the luminal membrane of intestinal cells. This is important as NaPiIlb (in lung) and Pit-2 (in brain) are both linked to severe inherited disorders, pulmonary alveolar microlithiasis and basal ganglia calcification, and it is unclear whether pharmacological inhibition could trigger similar symptoms. In healthy rats, EOS789 increased fecal phosphate excretion while reducing renal phosphate excretion (as part of the normal renal compensation). Importantly, EOS 789 was also able at the highest dose used to reduce and even normalize serum phosphate, FGF23 and PTH levels in rats with adenine-induced renal impairment. Similarly, EOS789 maintained low serum phosphate and FGF23 in a Thy.1.1 nephritis rat model. Since EOS789 inhibits in vitro several phosphate transporters present in the intestine, Tsuboi and colleagues compared the pantransporter inhibitor EOS789 to a specific NaPi-Ilb inhibitor and found that EOS was more effective in lowering serum phosphate levels and increasing fecal phosphate content than the NaPi-Ilb inhibitor. This may indicate that EOS789 targets also in vivo PiT1, PiT2, and NaPi-llb and that the PiT-transporters substantially contribute to intestinal phosphate absorption under these conditions. Alternatively, the two inhibitors may have different potency and halflife times and that EOS789 might have broader efficacy. Nevertheless, the effect of EOS789 on serum phosphate, FGF23 and PTH was importantly associated with lower aortic calcifications and better preservation of kidney function and less fibrosis. The drug also associated with lower serum calcium levels which may be caused by intestinal phosphate retention leading to calcium-phosphate complexes impairing intestinal calcium absorption. 
This study has several implications and asks important questions. First, previous studies in mouse models have indicated that NaPi-Ilb is the predominant phosphate transporter. The current study now suggests that other transporters from the Pit family may also be important. Of note, these seemingly conflicting data have been obtained in different species, mouse, rats, and humans. We may need to be careful with the choice of animal models and the translation of results from rodent models to the human condition. Second, what is the contribution of different phosphate transporters to overall intestinal phosphate absorption and how does this compare to paracellular phosphate absorption ? This may look like a purely academic question but may lead to a concept where transport inhibitors such as EOS789 are combined with phosphate binders. This would reduce bioavailable phosphate, decrease paracellular phosphate fluxes and at the same time block transcellular phosphate transport. The latter may be important as it has been suggested that phosphate binders stimulate expression of intestinal phosphate transporters. Indeed, the NoPhos study combining nicotinamide and phosphate binders is already testing this avenue. Third, the timing of therapies (predialysis vs dialysis) and the choice of targets may be important. While novel drugs increasing renal phosphate clearance through inhibition of renal phosphate transporters may be powerful in patients with residual kidney function, the intestine must be targeted in patients without kidney function. Also, combinations of intestinal and renal transport inhibitors should be considered.

The study by Tsuboi together with other studies testing tenapanor, nicotinamide alone or in combination with phosphate binders, and also the novel inhibitors of renal phosphate reabsorption by $\mathrm{NaPi}$-Ila demonstrate that novel approaches to hyperphosphatemia in predialysis and dialysis patients hold some promise for new therapies. Yet, our understanding of the molecular mechanisms underlying intestinal phosphate absorption in humans is very incomplete, the (long-term) efficacy of these novel therapies to lower serum phosphate levels in 
patients and most importantly to reduce morbidity and mortality in these patients has to be shown.

\section{Disclosure}

C.A.Wagner has received honoraria from Medice, AstraZeneca, and Bayer AG. Work in the laboratory of the author has been supported by the Swiss National Science Foundation.

\section{Figure legend}

Schematic model of intestinal phosphate absorption involving several phosphate transporters and the paracellular pathway. Various therapies either already clinically used and in preclinical tests target distinct pathways and mechanisms.

\section{References}

1. Dhayat NA, Ackermann D, Pruijm M, et al. Fibroblast growth factor 23 and markers of mineral metabolism in individuals with preserved renal function. Kidney Int 2016; 90: 648-657.

2. Hu MC, Shiizaki K, Kuro-o M, et al. Fibroblast growth factor 23 and Klotho: physiology and pathophysiology of an endocrine network of mineral metabolism. Annu Rev Physiol 2013; 75: 503-533.

3. Vervloet MG, Sezer S, Massy ZA, et al. The role of phosphate in kidney disease. Nat Rev Nephrol 2017; 13: 27-38.

4. Hernando N, Wagner CA. Mechanisms and Regulation of Intestinal Phosphate Absorption. Compr Physiol 2018; 8: 1065-1090.

5. Hernando N, Myakala K, Simona F, et al. Intestinal Depletion of NaPiIlb/Slc34a2 in Mice: Renal and Hormonal Adaptation. J Bone Miner Res 2015; 30: 1925-1937. 
6. Knopfel T, Pastor-Arroyo EM, Schnitzbauer $U$, et al. The intestinal phosphate transporter NaPi-llb (Slc34a2) is required to protect bone during dietary phosphate restriction. Sci Rep 2017; 7: 11018.

7. Cozzolino $M$, Ketteler $M$, Wagner CA. An expert update on novel therapeutic targets for hyperphosphatemia in chronic kidney disease: preclinical and clinical innovations. Expert Opin Ther Targets 2020: 1-12.

8. King AJ, Siegel M, He Y, et al. Inhibition of sodium/hydrogen exchanger 3 in the gastrointestinal tract by tenapanor reduces paracellular phosphate permeability. Sci Trans/ Med 2018; 10.

9. Tsuboi $Y$, Ohtomo S, Ichida $Y$, et al. EOS789, a novel inhibitor of multiphosphate transporters, is effective for the treatment of chronic kidney disease-mineral bone disorder. Kidney Int 2020. 


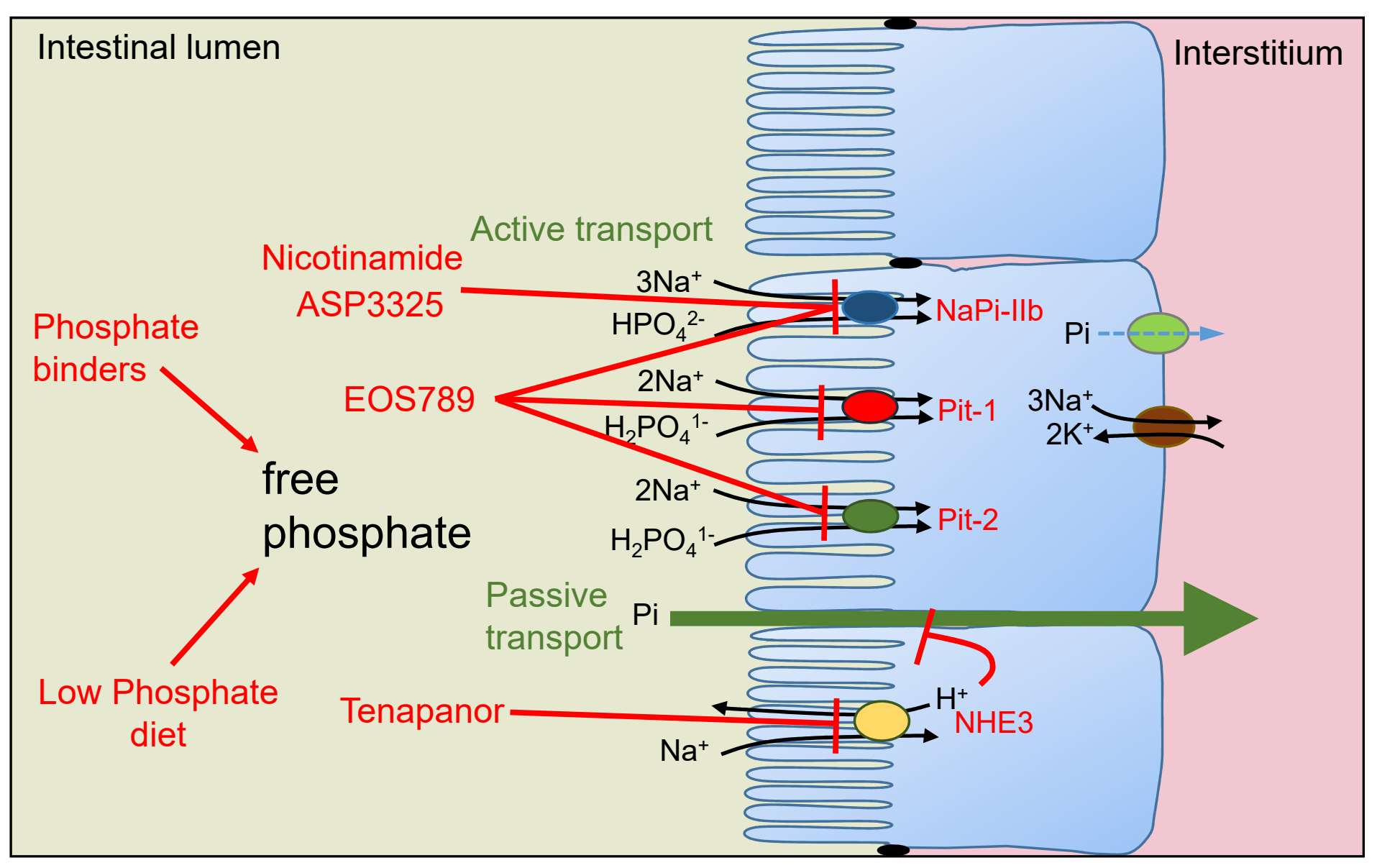

